EP58 PROCEDURAL AND FUNCTIONAL OUTCOMES FOR ASPIRATION THROMBECTOMY IN ACUTE ISCHEMIC STROKE PATIENTS WITH DIFFERING CLOT DWELL TIMES: A SUBSET ANALYSIS FROM THE COMPLETE REGISTRY

${ }^{1} \mathrm{O}$ Zaidat, ${ }^{2}$ AE Hassan, ${ }^{1} \mathrm{~S}$ Abdul Kareem, ${ }^{3} \mathrm{JT}$ Fifi. ${ }^{1}$ Mercy Health St. Vincent Medical Center, Toledo, OH; ${ }^{2}$ University of Texas Rio Grande Valley, Valley Baptist Medical Center, Harlingen, TX; ${ }^{3}$ cahn School of Medicine at Mount Sinai, New York, NY, USA

\subsection{6/neurintsurg-2021-ESMINT.57}

Introduction Longer clot dwell times following acute ischemic stroke (AIS) are potentially associated with poorer outcomes in patients treated with aspiration thrombectomy (AT).

Aim of the Study Evaluate the safety and efficacy of AT in AIS across differing clot dwell times.

Methods A subset analysis from a global prospective registry of adults with AIS (COMPLETE) was performed to evaluate impact of clot dwell time on functional outcomes following AT with the Penumbra System. Inclusion criteria were M1 occlusion, witnessed stroke, baseline mTICI $0-2 \mathrm{a}$, and onset to puncture time of $0-24$ hours.Patients with multiple emboli, proximal stenosis, or tandem lesions were excluded.

Results Among 148 patients included (mean age 67.4, 57\% female), 67 had clots $<=3$ hours and 81 had clots $>3$ hours. Older clots had lower ASPECTS (median 8.0 [IQR 6.0, 9.0] versus $9.0[8.0,10.0] \mathrm{p}=0.0003)$ and higher NIHSS (13.0, $[9.0,18.0]$ versus $16.0[11.0,20.0] \mathrm{p}=0.0005)$ at baseline. Older clots required more passes $(2.0[1.0,3.0]$ versus 1.0 $[1.0,2.0], \mathrm{p}=0.0066)$, and time to mTICI $2 \mathrm{~b}-3$ reperfusion ( median 27.0 [17.0, 42.0] versus 17.0 [13.0, 29.0] minutes, $\mathrm{p}=0.0094)$. Each hour increase in dwell time reduced odds of functional recovery (90-day mRS $0-2$ ) by $12 \%$ (OR 0.88 ; $95 \%$ CI $0.661,0.989 ; \mathrm{p}=0.0388)$ with no significant difference in mortality. Older clots were associated with more safety complications and longer hospital stays.

Conclusions Patients with longer clot dwell times were associated with more attempts and longer time to achieve reperfusion, more post-procedure complications, and lower likelihood of functional recovery.

Disclosure Osama O. Zaidat: Grant/research support from Genentech, Medtronic Neurovascular, Stryker. Consultant for Codman, Medtronic Neurovascular, National Institutes of Health StrokeNet, Penumbra, Stryker. Honoraria from Codman, Medtronic Neurovascular, Penumbra, Stryker. Serves as an expert witness. Ownership interest in Galaxy Therapeutics, Inc. Johanna T. Fifi: Grant/research support: Microvention, Penumbra, Stryker. Consultant: Microvention, Stryker. Other financial or material support: Ownership interest: Imperative Care. Ameer E. Hassan: Consultant/Speakers bureau: GE Healthcare, Genentech, Medtronic, Microvention, Penumbra, Stryker, Cerenovus, Viz.ai, Balt and Scientia

\section{EP59 SUCCESSFUL MECHANICAL THROMBECTOMY AFTER MICROSURGICAL CLIPPING OF A RUPTURED MIDDLE CEREBRAL ARTERY ANEURYSM}

M Boutchakova-Meyer, P Beyaz, C Roth, P Papanagiotou. Neuroradiology, Klinikum Bremen Mitte, Bremen, Germany

10.1136/neurintsurg-2021-ESMINT.58

In case of acute thrombotic occlusion of the parent artery after microsurgical aneurysm clipping an endovascular mechanical thrombectomy is an essential technique to achieve vascular recanalization. However a procedure-related rerupture risk should be considered, especially when using a stent retriever.

To demonstrate the feasibility and safety of mechanical thrombectomy for vessel occlusion after clipping of a ruptured aneurysm of the middle cerebral artery (MCA) trifurcation.

We report about a 62 year old woman who was brought to the emergency room after being found down at home. On admission she was somnolent but accessible with mild focal neurological deficits. A computed tomography of the brain revealed a right temporal hematoma with subarachnoid and intraventricular hemorrhage due to a ruptured saccular aneurysm of the MCA trifurcation. A surgical approach was considered best treatment option and microsurgical clipping was performed. Intraoperative microvascular Doppler depicted a spontaneous thrombus formation in M1-Segment and the patient was transferred to the angio-suite for a mechanical thrombectomy.

Cerebral angiography demonstrated occlusion of the M1Segment on the right site. After two failed contact aspiration thrombectomy attempts, a combination of stent retriever and aspiration was performed. A microcatheter was advanced into the M2-Segment and a Solitare device was deployed from the middle M2-Artery to M1-Segment.Postthrombectomy imaging demonstrated TICI $2 \mathrm{~b}$ recanalization. There were no procedure related complications, no perforation and no vasospasm. The treated aneurysm was completely obliterated.

Stent-retriever revascularization is a safe, feasible and effective treatment option for acute thrombotic occlusion of the parent artery after microsurgical aneurysm clipping.

\section{REFERENCES}

1. Konakondla $\mathbf{S}$, et al. Zero-delay mechanical thrombectomy for distal large vesse occlusion detected on intraoperative angiogram after microsurgical clipping of a posterior communicating artery aneurysm: value of hybrid operating room. World Neurosurg 2018 Nov;119:278-281.

2. Jabbarli, et al. Aneurysm remnant after clipping: the risks and consequences. Journal of Neurosurgery JNS 2016;125(5):1249-1255.

3. Zibold F, et al. Aneurysms in the target vessels of stroke patients subjected to mechanical thrombectomy: prevalence and impact on treatment. I Neurointerv Surg 2016 Oct;8(10):1016-20.

4. Zhou $\mathrm{T}$, et al. Endovascular thrombectomy for large-vessel occlusion strokes with preexisting intracranial aneurysms. Cardiovasc Intervent Radiol 2018 Sep;41 (9):1399-1403.

Disclosure Nothing to disclose

\section{EP60 ENDOVASCULAR THROMBECTOMY IN POSTERIOR CIRCULATION STROKE AT SAINT PETERSBURG VASCULAR CENTER}

${ }^{1}$ A Savello, ${ }^{2} \mathrm{~K}$ Orlov. ${ }^{1}$ Military Medical Academy of S.M. Kirov, Saint Petersburg; ${ }^{2}$ Federal Center for Brain Research and Neuro Technology, Fedral Biomedical Agency, Moscow, Russian Federation

\subsection{6/neurintsurg-2021-ESMINT.59}

We performed a retrospective study of 211 patients (128 male and 83 female, m.age 68 years) with acute LVO in posterior circulation. These patients with ischemic stroke underwent EVT in regional vascular centers.

Results In most cases (80.6\%), the clots were removed by thromboaspiration as the first-line reperfusion method, and in $12.8 \%$ of cases - using stent retrievers. Although the need to change the reperfusion method was higher with stent 
retrievers compared to thromboaspiration $(40.7 \%$ against $27.6 \%)$, there was no statistically relevant difference $(p=0,165)$. Thromboaspiration as the first-line reperfusion method was associated with more complete (mTICI 3, $74.1 \%$ ) and successful (mTICI 2b-3, 86.5\%) reperfusion by the end of the intervention. The use of stent retrievers as the first-line method led to less frequent complete $(55.6 \%)$ $(p=0,048)$ and successful $(66.6 \%) \quad(p=0,009)$ reperfusion. The efficiency of the reperfusion in posterior circulation depends on the first-line method and the number of passes. Thus, after the first pass with a stent retriever the level of successful $(p=0.04)$ and complete $(p=0.015)$ reperfusion is statistically lower than with thromboaspiration. SAVE technique also proved to be effective in achieving more successful $(p=0.015)$ and complete $(p=0.003)$ reperfusion comparing to stent retrievers. However, thrombectomy with a stent retriever as a second-line method of recanalization was used more often than thromboaspiration or SAVE. The second-line method was ineffective in $25.8 \%$ of cases, which required switching to the third method of reperfusion. This figure is comparable to the need to switch to the second-line reperfusion method in 29.4\% of cases.

Disclosure Nothing to disclose

\section{EP61 DIRECT CAROTID PUNCTURE FOR MECHANICAL THROMBECTOMY IN ACUTE ISCHAEMIC STROKE: A SINGLE CENTRE EXPERIENCE AND REVIEW OF THE LITERATURE}

JCY Chan, IHW Cheung, YLE Chu, R Lee. Department of Radiology, Queen Mary Hospital, Hong Kong, Hong Kong

\subsection{6/neurintsurg-2021-ESMINT.60}

Introduction Direct carotid puncture (DCP) as a bailout or primary vascular access technique for endovascular thrombectomy (EVT) has been sporadically described in the literature. The collective procedural risk profile and therapeutic outcomes remain unclear.

Objectives and Aims To establish the efficacy and safety profile of DCP.

Method We reviewed our prospectively maintained singlecentre database of patients admitted for acute ischaemic stroke (AIS) who underwent EVT. 11 patients treated by DCP approach were identified. We also conducted a literature review on published cases of EVT performed via DCP.

Results 9 studies with a total of 106 cases (our data included) were reviewed. Initial NIHSS score ranges from 2 to 31 (average 17.1). DCP access was successful in 92.5\%. Among this, $86 \%$ achieved satisfactory recanalization (mTICI $\geq 2 \mathrm{~b}$ ). Average post-procedural $\mathrm{mRS}$ is 3.8. Carotid access sites were managed with closure devices in $76.6 \%$, with Angioseal being the most commonly deployed device. Haemostasis was achieved by manual compression or combined method in $22.2 \%$ and $5.1 \%$ of the cases respectively.

Carotid access site-related complications were encountered in 19 cases (17.9\%). These include puncture site haematoma $(n=12)$, non-flow-dependent carotid artery dissection $(n=4)$, access site pseudoaneurysm $(n=2)$ and retinal artery occlusion $(n=1) .4$ cases required further intervention(3.8\%). No mortality related to access site complication was reported.

Conclusion Direct carotid puncture is an effective and generally safe approach for EVT, with major access-site related complications seen in $<5 \%$ of the cases. It should be considered as a bailout technique or primary access approach in selected cases.

\section{REFERENCES}

1. Akpinar CK, et al. Direct common carotid artery puncture: rescue mechanica thrombectomy strategy in acute ischemic stroke. Neurointervention 2020;15 (2):60-66.

2. Cord BJ, et al. Direct carotid puncture for mechanical thrombectomy in acute ischemic stroke patients with prohibitive vascular access. I Neurosurg 2020:1-11.

3. Miszczuk M, et al. Direct puncture of the carotid artery as a bailout vascular access technique for mechanical thrombectomy in acute ischemic stroke-the revival of an old technique in a modern setting. Neuroradiology 2021;63(2):275-283.

Disclosure Nothing to disclose

\section{EP62 ENDOVASCULAR TRANSCAROTID ARTERY REVASCULARIZATION USING THE WALRUS BALLOON GUIDE CATHETER: SAFETY AND FEASIBILITY FROM MULTICENTER EXPERIENCE}

${ }^{1} \mathrm{M}$ Salem, ${ }^{2} \mathrm{C}$ Griessenauer, ${ }^{1} \mathrm{~S}$ Kvint, ${ }^{3} \mathrm{~A}$ Baig, ${ }^{3} \mathrm{~A}$ Monteiro, ${ }^{4} \mathrm{G}$ Cortez, ${ }^{5} \mathrm{~A}$ Kuhn, ${ }^{1} \mathrm{O}$ Choudhri, ${ }^{2} \mathrm{O}$ Goren, ${ }^{2} \mathrm{~S}$ Dalal, ${ }^{6} \mathrm{P}$ Jabbour, ${ }^{7} \mathrm{RM}$ Starke, ${ }^{5} \mathrm{~A}$ Puri, ${ }^{4} \mathrm{R}$ Hanel, ${ }^{3} \mathrm{El}$ Levy, ${ }^{3}$ A Siddiqui, ${ }^{1} \mathrm{~J}-\mathrm{K}$ Burkhardt*. ${ }^{1}$ Penn Medicine, Hospital of the University of Pennsylvania, Philadelphia; ${ }^{2}$ Geisinger Medical Center, Danville, PA; ${ }^{3}$ University at Buffalo, State University of New York, Buffalo, NY; ${ }^{4}$ Baptist Stroke and Cerebrovascular Center, Jacksonville, $F L$; ${ }^{5}$ UMASS, 55 Lake Avenue North, Worcester, MA; ${ }^{6}$ Jefferson Health, Philadelphia, PA; 7 University of Miami, Miami, FL, United States

\subsection{6/neurintsurg-2021-ESMINT.61}

Introduction The Walrus Balloon Guide Catheter (BGC) is a new generation of BGC, designed to bypass limitations of conventional BGCs in mechanical thrombectomy.

Objectives To analyze the Walrus BGC for cervical carotid disease (CCD) using the endovascular transcarotid artery revascularization (eTCAR) technique.

Aims Safety and feasibility from a multicenter experience.

Methods Retrospective analysis of prospectively-maintained multicenter datasets

Results 105 patients with high-grade CCD (median carotid stenosis of $83 \%$ ) were included. Navigating the Walrus BGC in the common carotid artery (CCA) was successful in all cases despite type 3/bovine arch anatomy in $26.7 \%$. Emergent treatment for cervical tandem occlusion along with mechanical thrombectomy for acute ischemic stroke was performed in $35.2 \%$ of the cases, with successful recanalization rate (TICI $2 \mathrm{~b} / 3$ ) of $81.1 \%$. Utilizing femoral access in $81.9 \%$ of the patients, carotid stenting was performed in all cases except 4 (angioplasty only); adjunct angioplasty and distal protection devices were used in $86.7 \%$ and in $53.4 \%$ of the cases, respectively. Flow arrest was utilized in the majority of the procedures $(87.6 \%)$, with successful stent deployment achieved in all cases. No major ischemic or Walrus BGC related complications, myocardial infarction or mortality was encountered. Last follow-up mRS of $0-2$ was $70.5 \%$ overall and $98.2 \%$ in elective eTCAR.

Conclusion We present a large multicenter experience of eTCAR technique utilizing the Walrus BGC. In all cases elective or emergent carotid stenting was successful with proximal flow arrest or flow reversal with or without distal protection device with favorable safety profile on follow-up.

Disclosure Jan-Karl Burkhardt, consultant for Q`Apel Medical, Longeviti Neuro Solutions 